\title{
A COMMUTATIVE ALGEBRAIC APPROACH TO THE FITTING PROBLEM
}

\author{
ŞTEFAN O. TOHǍNEANU \\ (Communicated by Irena Peeva)
}

\begin{abstract}
Given a finite set of points $\Gamma$ in $\mathbb{P}^{k-1}$ not all contained in a hyperplane, the "fitting problem" asks what is the maximum number $h y p(\Gamma)$ of these points that can fit in some hyperplane and what is (are) the equation(s) of such hyperplane(s)? If $\Gamma$ has the property that any $k-1$ of its points span a hyperplane, then $\operatorname{hyp}(\Gamma)=\operatorname{nil}(I)+k-2$, where $\operatorname{nil}(I)$ is the index of nilpotency of an ideal constructed from the homogeneous coordinates of the points of $\Gamma$. Note that in $\mathbb{P}^{2}$ any two points span a line, and we find that the maximum number of collinear points of any given set of points $\Gamma \subset \mathbb{P}^{2}$ equals the index of nilpotency of the corresponding ideal, plus one.
\end{abstract}

\section{INTRODUCTION}

Let $\mathbb{K}$ be any field, and let $\Gamma \subseteq \mathbb{P}_{\mathbb{K}}^{k-1}$ be a finite reduced set of points, not all contained in a hyperplane. Let $h y p(\Gamma)$ be the maximum number of points of $\Gamma$ contained in some hyperplane.

Computationally, the "fitting problem" (or "exact fitting problem") asks for effective methods or algorithms to compute this number and to find the equation of the hyperplane. If $\Gamma$ is $(k-2)$-generic (i.e., any $k-1$ of the points span a hyperplane) 1 by [3] (or [8, Algorithm MinN1]), the problem of finding the hyperplane can be solved in $O\left(|\Gamma|^{k-1}\right)$ time. If one knows $h y p(\Gamma)$, in [8, Corollary 3.3], is presented an algorithm that finds the points in this hyperplane in $O\left(\min \left\{\frac{|\Gamma|^{k-1}}{h y p(\Gamma)^{k-2}} \log \left(\frac{|\Gamma|}{h y p(\Gamma)}\right),|\Gamma|^{k-1}\right\}\right)$ time.

The fitting problem is directly connected with the computation of the minimum distance $d$ of the equivalence class of linear codes with generating matrix having as columns the coordinates of the points; the points can be placed as columns in any order, and homogeneous coordinates are the same up to multiplication by a nonzero constant 2 The connection is that $d=|\Gamma|-h y p(\Gamma)$. With this, [6], 9], 12], [13], 14, or [15] shows that the minimum distance gives bounds for homological invariants of zero-dimensional projective schemes. We should also mention that the hyperplanes that contain $h y p(\Gamma)$ points of $\Gamma$ are in one-to-one correspondence with the (projective) codewords of minimum weight of the class of linear codes constructed from $\Gamma$ : the coefficients of the linear form defining such a hyperplane

Received by the editors March 1, 2012 and, in revised form, March 22, 2012.

2010 Mathematics Subject Classification. Primary 52C35; Secondary 13P25, 13P20.

Key words and phrases. Index of nilpotency, fat points, minimum distance.

${ }^{1}$ In [3] and [8], the set of points $\Gamma$ is in the affine space $\mathbb{A}^{k-1}$. Nevertheless we can embed it into $\mathbb{P}^{k-1}$ by adding to each point of $\Gamma$ an extra coordinate that equals 1 .

${ }^{2}$ For background on linear codes we recommend [1]. 
are the coefficients of the linear combination of the rows of the generating matrix of the linear code that will give the codeword of minimum weight. Therefore, Lemma 2.2 in 13 allows us to determine these hyperplanes by finding the minimal primes of a certain ideal, which is very simple once we know hyp $(\Gamma)$.

In this paper we link $h y p(\Gamma)$ to the index of nilpotency of an ideal generated by products of linear forms. We can do this if $\Gamma \subset \mathbb{P}^{k-1}$ is $(k-2)$-generic. This restriction does not occur if $k-1=2$, and therefore we give a new interpretation to the fitting problem of any set of points in the plane.

The article is structured as follows. In the next section we generalize a result of Schenck (11], Lemma 3.1, together with Lemma 3.2) known so far to be true only for $\mathbb{P}^{2}$ : let $\mathcal{A}$ be an arrangement of $n$ lines in $\mathbb{P}^{2}$, defined by the linear forms $L_{1}, \ldots, L_{n} \in R:=\mathbb{K}[x, y, z]$. If $\operatorname{Sing}(\mathcal{A}) \subset \mathbb{P}^{2}$ denotes the singular locus of $\mathcal{A}$ (i.e., the set of intersection points of the lines in $\mathcal{A}$ ), then, ideal-theoretically, the following is true:

$$
\left\langle L_{2} L_{3} \cdots L_{n}, L_{1} L_{3} \cdots L_{n}, \ldots, L_{1} L_{2} \cdots L_{n-1}\right\rangle=\bigcap_{P \in \operatorname{Sing}(\mathcal{A})} I(P)^{n_{P}-1},
$$

where $I(P) \subset R$ is the ideal of the point $P$ and $n_{P}$ is the number of lines of $\mathcal{A}$ intersecting at $P$. This result, along with the results of Davis and Geramita (see 2]), was essential in showing that the Orlik-Terao algebra of an arrangement of lines in $\mathbb{P}^{2}$ is the homogeneous coordinate ring associated to a nef on the blowup of $\mathbb{P}^{2}$ at the singularities of the arrangement (see [1] for more details). In the final part we discuss the index of nilpotency of fat points and we prove our main result, Theorem 3.2

The goal of this paper is to obtain an understanding of the fitting problem from a commutative algebraic point of view. Therefore our effort is directed towards presenting this abstract approach with as many details as possible for a nonspecialist, leaving the analysis of the efficiency of the possible algorithms that may be created from our exposition to the experts in the field.

\section{A fat point SCHEME CONSTRUCTED FROM HYPERPLANE ARRANGEMENTS}

Let $\mathcal{A}$ be a central essential hyperplane arrangement in $V=\mathbb{K}^{k}$. Suppose $\mathcal{A}=\left\{H_{1}, \ldots, H_{n}\right\}$ and each $H_{i}$ is the vanishing of a linear form $L_{i} \in \operatorname{Sym}\left(V^{*}\right)=$ $\mathbb{K}\left[x_{1}, \ldots, x_{k}\right]$. "Central" means that all the hyperplanes of $\mathcal{A}$ pass through the origin, and "essential" means that the rank of $\mathcal{A}$ is $k$ (i.e., $\operatorname{codim}\left(H_{1} \cap \cdots \cap H_{n}\right)=k$ ). For more details and background on hyperplane arrangements, we recommend [10].

To any hyperplane arrangement one can associate the lattice of intersection $L(\mathcal{A})$, which is a lattice built on the intersections of hyperplanes, with levels $L_{j}(\mathcal{A})$ : $X=H_{i_{1}} \cap \cdots \cap H_{i_{s}} \in L_{j}(\mathcal{A})$ if and only if $\operatorname{codim}(X)=j . X$ is called a flat of rank $j$, and $s$, which is the number of hyperplanes that contain $X$, will be denoted by $\nu(X)$. Flats of rank $k-1$ are called coatoms.

We are interested in a special class of hyperplane arrangements. A central essential hyperplane arrangement $\mathcal{A} \subset \mathbb{K}^{k}$ will be called $k-2$ generic if and only if any $k-1$ of the linear forms $L_{i}$ defining the hyperplanes of $\mathcal{A}$ are linearly independent. Observe that all arrangements of rank 3 are $k-2$ generic (unless they are multiarrangements). 
For a hyperplane arrangement $\mathcal{A} \subset \mathbb{K}^{k}$, define

$$
I_{j}(\mathcal{A})=\left\langle\left\{L_{i_{1}} \cdots L_{i_{j}}: 1 \leq i_{1}<\cdots<i_{j} \leq n\right\}\right\rangle \subset R:=\mathbb{K}\left[x_{1}, \ldots, x_{k}\right],
$$

the ideal generated by all the distinct $j$ products of the linear forms defining the hyperplanes of $\mathcal{A}$.

With all of these we can generalize the result of Schenck to arbitrary rank. But first, one more definition. Let $I$ be a homogeneous ideal in the polynomial ring $R:=\mathbb{K}\left[x_{1}, \ldots, x_{k}\right]$. The saturation of $I$ is the ideal

$$
I^{\text {sat }}=\left\{f \in R \mid f \in I:\left\langle x_{1}, \ldots, x_{k}\right\rangle^{n(f)} \text { for some } n(f)\right\} .
$$

Proposition 2.1. Let $\mathcal{A}$ be a central essential $k-2$ generic arrangement of $n$ hyperplanes in $\mathbb{K}^{k}$. Then

$$
I_{n-k+2}(\mathcal{A})=\bigcap_{X \in L_{k-1}(\mathcal{A})} I(X)^{\nu(X)-k+2} .
$$

Proof. Following the same considerations as in Section 4 in [13], any minimal prime of the ideal $I_{n-k+2}(\mathcal{A})$ is of the form

$$
\left\langle L_{i_{1}}, \ldots, L_{i_{k-1}}\right\rangle \text {. }
$$

So all the minimal primes have codimension exactly $k-1$ (since $\mathcal{A}$ is $k-2$ generic), and they are the ideals of the coatoms of $\mathcal{A}$.

From this we get: 1) the codimension of $I_{n-k+2}(\mathcal{A})$ is $k-1$, and 2) $I_{n-k+2}(\mathcal{A})$ might have an embedded prime, the irrelevant ideal $\left\langle x_{1}, \ldots, x_{k}\right\rangle$. So the primary decomposition of $I_{n-k+2}(\mathcal{A})$ is

$$
I_{n-k+2}(\mathcal{A})=Q_{1} \cap \cdots \cap Q_{s} \cap J,
$$

where $Q_{i}$ are primary ideals of codimension $k-1$ and $J$ is a $\left\langle x_{1}, \ldots, x_{k}\right\rangle$-primary ideal of codimension $k$. Since for any two ideals $A, B \subset R$ we have $(A \cap B)^{\text {sat }}=$ $A^{\text {sat }} \cap B^{\text {sat }}$ and because $J^{\text {sat }}=R$, we obtain that

$$
I_{n-k+2}(\mathcal{A})^{s a t}=Q_{1} \cap \cdots \cap Q_{s} .
$$

Here we used the fact that $Q_{i}$ are primary ideals of codimension $k-1$ and therefore $\left(Q_{i}\right)^{\text {sat }}=Q_{i}$. If $f \in\left(Q_{i}\right)^{\text {sat }}$ but $f \notin Q_{i}$, then $\left\langle x_{1}, \ldots, x_{k}\right\rangle^{n(f)} \cdot f \subseteq Q_{i}$, for some positive integer $n(f)$. From the definition of the primary ideals, we obtain that $\left\langle x_{1}, \ldots, x_{k}\right\rangle^{u} \subseteq Q_{i}$, for some power $u>0$, which is in contradiction with $\operatorname{codim}\left(Q_{i}\right)=k-1$.

To prove our assertion, first we show that $I_{n-k+2}(\mathcal{A})^{\text {sat }}$ has the primary decomposition described in the statement by localizations of $I_{n-k+2}(\mathcal{A})$ at each of its minimal primes. Then we show that $I_{n-k+2}(\mathcal{A})=I_{n-k+2}(\mathcal{A})^{\text {sat }}$.

Let $X \in L_{k-1}(\mathcal{A})$ be a coatom. Denote $\nu(X)=m$, and assume that

$$
X=H_{1} \cap \cdots \cap H_{m},
$$

with $I(X)=\left\langle x_{1}, \ldots, x_{k-1}\right\rangle \subset R:=\mathbb{K}\left[x_{1}, \ldots, x_{k}\right]$.

If we localize $R$ at $I(X)$, we have that $L_{m+1}, \ldots, L_{n}$ are invertible elements and therefore

$$
I_{n-k+2}(\mathcal{A}) R_{I(X)}=\left\langle\left\{L_{i_{1}} \cdots L_{i_{m-k+2}}: 1 \leq i_{1}<\cdots<i_{j} \leq m\right\}\right\rangle R_{I(X)} .
$$

Now consider the linear code with generating matrix $A$ given by the coefficients of the linear forms $L_{1}, \ldots, L_{m}$. These are linear forms in variables $x_{1}, \ldots, x_{k-1}$. So this linear code has length $m$ and dimension $k-1$. Since any $k-1$ of these linear 
forms are linearly independent, the maximum number of columns of $A$ that span a $k-2$-dimensional vector space is $k-2$. So, by [15, Remark 2.3], the minimum distance of this code is

$$
d=m-(k-2)=m-k+2 .
$$

From [13, Theorem 3.1], we have that

$$
\left\langle\left\{L_{i_{1}} \cdots L_{i_{m-k+2}}: 1 \leq i_{1}<\cdots<i_{j} \leq m\right\}\right\rangle=\left\langle x_{1}, \ldots, x_{k-1}\right\rangle^{m-k+2} .
$$

So we get that

$$
I_{n-k+2}(\mathcal{A}) R_{I(X)}=I(X)^{\nu(X)-k+2} R_{I(X)},
$$

for all the minimal primes (which are the ideals $I(X)$ of all the coatoms $X$ ) of $I_{n-k+2}(\mathcal{A})$. This means that the primary decomposition of $I_{n-k+2}(\mathcal{A})^{s a t}$ is the one desired.

Any $I_{i}(\mathcal{A})$ can be generated by the maximal minors of a certain matrix. Let $M$ be an $i \times n$ matrix with entries in $\mathbb{K}$ such that all the $i \times i$ minors are nonzero. There exists such a matrix since, by the way it is defined, the set of all these matrices is an open Zariski set. Then the maximal minors of the $i \times n$ matrix with entries in $R_{1}$

$$
N=M \cdot\left[\begin{array}{cccc}
L_{1} & 0 & \cdots & 0 \\
0 & L_{2} & \cdots & 0 \\
\vdots & \vdots & & \vdots \\
0 & 0 & \cdots & L_{n}
\end{array}\right]
$$

are the generators of $I_{i}(\mathcal{A})$.

When $i=n-k+2$ and $\mathcal{A}$ is $k-2$ generic, the codimension of $I_{i}(\mathcal{A})$ is exactly $(i-i+1)(n-i+1)=k-1$. So $R / I_{n-k+2}(\mathcal{A})$ is a determinantal ring which is known to be Cohen-Macaulay (4, Theorem 18.18).

So, the projective dimension of $R / I_{n-k+2}(\mathcal{A})$ is

$$
\operatorname{pd}\left(R / I_{n-k+2}(\mathcal{A})\right)=\operatorname{codim}\left(I_{n-k+2}(\mathcal{A})\right)=k-1 .
$$

This means that $\operatorname{Ext}^{k}\left(R / I_{n-k+2}(\mathcal{A}), R\right)=0$, and this gives that $I_{n-k+2}(\mathcal{A})$ cannot have an associated prime of codimension $k$. Therefore, $I_{n-k+2}(\mathcal{A})=I_{n-k+2}(\mathcal{A})^{s a t}$.

For rank 3 arrangements, 11, Lemma 3.2], presents the graded minimal free resolution for $I_{n-1}(\mathcal{A})$. More generally, from the map $R^{n} \longrightarrow R^{i}$ with matrix $N$ seen in the proof above, we get a complex of $R$-modules, known as the EagonNorthcott complex ([5], Chapter A2H, covers in full the details of this complex). If $\operatorname{depth}\left(I_{i}(\mathcal{A}), R\right)=n-i+1$, then this complex is exact and therefore it provides a free resolution for $R / I_{i}(\mathcal{A})$.

In our instance, $i=n-k+2$, and since $R / I_{n-k+2}(\mathcal{A})$ is Cohen-Macaulay, $k-1$ is the smallest integer $r$ such that $\operatorname{Ext}^{r}\left(R / I_{n-k+2}(\mathcal{A}), R\right) \neq 0$. By [4, Proposition 18.4],

$$
\operatorname{depth}\left(I_{n-k+2}(\mathcal{A}), R\right)=k-1=n-(n-k+2)+1,
$$

and we have the exactness of the Eagon-Northcott complex for the $R$-module $R / I_{n-k+2}(\mathcal{A})$. 


\section{THE FITTING PROBLEM AND THE INDEX OF NILPOTENCY}

Let $I$ be an ideal in $R=\mathbb{K}\left[x_{1}, \ldots, x_{k}\right]$. The index of nilpotency of $I$, denoted $\operatorname{nil}(I)$, is the smallest integer $s$ such that

$$
(\sqrt{I})^{s} \subseteq I .
$$

For a nice exposition about this invariant we recommend [16, Chapter 9.2].

The next result determines the index of nilpotency of the ideal of a fat point scheme in $\mathbb{P}^{k-1}$.

Proposition 3.1. Let $Z=m_{1} P_{1}+\cdots+m_{n} P_{n}, m_{i} \geq 1$, be a fat point scheme in $\mathbb{P}_{\mathbb{K}}^{k-1}$. If $I_{Z} \subset R=\mathbb{K}\left[x_{1}, \ldots, x_{k}\right]$ is the ideal of $Z$, then

$$
\operatorname{nil}\left(I_{Z}\right)=\max \left\{m_{1}, \ldots, m_{n}\right\} .
$$

Proof. Let $X=\left\{P_{1}, \ldots, P_{n}\right\} \subset \mathbb{P}^{k-1}$ be the support of $Z$. Let $I_{X} \subset R$ be the ideal of $X$. Then

$$
\sqrt{I_{Z}}=I_{X}
$$

Let $s=n i l\left(I_{Z}\right)$ and $m=\max \left\{m_{1}, \ldots, m_{n}\right\}$. Denote by $I_{P_{i}}$ the ideal of the point $P_{i}$. Suppose that $m=m_{1}$ and that $P_{1}=[0, \ldots, 0,1]$.

We have that

$$
I_{Z}=I_{P_{1}}^{m_{1}} \cap \cdots \cap I_{P_{n}}^{m_{n}}
$$

and

$$
I_{X}=I_{P_{1}} \cap \cdots \cap I_{P_{n}} .
$$

Obviously, $I_{X}^{m} \subseteq I_{Z}$. Therefore

$$
m \geq s \text {. }
$$

Suppose $m \geq 2$. Otherwise, $I_{Z}=I_{X}$, and therefore $\operatorname{nil}\left(I_{Z}\right)=1=m$.

Also, assume that $s \leq m-1$, and let $f \in I_{X}$ be such that $f \notin I_{P_{1}}^{2}$. There must exist such an element; otherwise $I_{P_{1}}^{2}$ would become a primary component of $I_{X}$, which contradicts that $I_{X}$ is a radical ideal.

Since $I_{X}^{s} \subseteq I_{Z}$, then

$$
f^{m-1} \in I_{P_{1}}^{m} .
$$

Because $f \in I_{P_{1}}-I_{P_{1}}^{2}$, and since $I_{P_{1}}=\left\langle x_{1}, \ldots, x_{k-1}\right\rangle$, we have that

$$
f=x_{1} g_{1}+x_{2} g_{2}+\cdots+x_{k-1} g_{k-1},
$$

with at least one of the polynomials $g_{i}$ not in $I_{P_{1}}$.

If $\operatorname{deg}(f)=d+1$, we can assume that

$$
f=\ell x_{k}^{d}+g,
$$

where $\ell$ is a linear form in variables $x_{1}, \ldots, x_{k-1}$, and $g \in I_{P_{1}}^{2}$.

Then

$$
f^{m-1}=\ell^{m-1} x_{k}^{d(m-1)}+h,
$$

where $h=\sum_{b=1}^{m-1}\left(\begin{array}{c}m-1 \\ b\end{array}\right) \ell^{m-1-b} g^{b} x_{k}^{d(m-1-b)}$. By the way we constructed $\ell$ and $g$,

$$
\ell^{m-1-b} g^{b} \in I_{P_{1}}^{m-1+b},
$$

and since $b \geq 1$, we have that the polynomial $h \in I_{P_{1}}^{m}$. 
We obtain $\ell^{m-1} x_{k}^{d(m-1)} \in I_{P_{1}}^{m}$, which is a contradiction: the leading monomial of $\ell^{m-1} x_{k}^{d(m-1)}$ under any monomial order $>$ with $x_{1}>\cdots>x_{k-1}>x_{k}$ is $x_{i}^{m-1} x_{k}^{d(m-1)}$ for some $1 \leq i \leq k-1$ and should belong to the (monomial) ideal $\left\langle x_{1}, \ldots, x_{k-1}\right\rangle^{m}$.

So $s \geq m$, and therefore $s=m$.

Now we can put together the two propositions to obtain the main result of this note. First, to a finite set of $n$ points $\Gamma \subset \mathbb{P}^{k-1}$, not all on a hyperplane, we can associate the central essential (dual) arrangement of $n$ hyperplanes $\mathcal{A}_{\Gamma} \subset \mathbb{K}^{k}$ defined by the vanishing of the linear forms with coefficients the coordinates of the points of $\Gamma$.

Theorem 3.2. Let $\Gamma \subset \mathbb{P}_{\mathbb{K}}^{k-1}$ be a finite $(k-2)$-generic set of $n$ points, not all contained in a hyperplane. Then

$$
\operatorname{hyp}(\Gamma)=\operatorname{nil}\left(I_{n-k+2}\left(\mathcal{A}_{\Gamma}\right)\right)+k-2 .
$$

Proof. If $h y p(\Gamma)$ number of points lie on a hyperplane of equation $a_{1} x_{1}+\cdots+a_{k} x_{k}=$ 0 , then, dually, the corresponding hyperplanes in $\mathcal{A}_{\Gamma}$ will intersect at the coatom $\left[a_{1}, \ldots, a_{k}\right]$. So

$$
\operatorname{hyp}(\Gamma)=\max \left\{\nu(X): X \in L_{k-1}\left(\mathcal{A}_{\Gamma}\right)\right\} .
$$

An immediate application of Proposition 3.1 to Proposition 2.1 gives the result.

Example 3.3. We end with a simple example. Let $P_{1}=(1,0), P_{2}=(1,1), P_{3}=$ $(3,-1), P_{4}=(-3,2)$ be four points in the real plane. Find the maximum number of collinear points and the equation(s) of the line(s) where they are positioned.

First we projectivize the problem by embedding the affine real plane into $\mathbb{P}^{2}$. So we add the extra coordinate $z=1$ to all the points to get

$$
\Gamma=\left\{Q_{1}=[1,0,1], Q_{2}=[1,1,1], Q_{3}=[3,-1,1], Q_{4}=[-3,2,1]\right\} \subset \mathbb{P}^{2} .
$$

To find $\operatorname{hyp}(\Gamma)$, we create $\mathcal{A}_{\Gamma}$ defined by the linear forms

$$
L_{1}=x+z, L_{2}=x+y+z, L_{3}=3 x-y+z, L_{4}=-3 x+2 y+z,
$$

and build

$$
I_{3}\left(\mathcal{A}_{\Gamma}\right)=\left\langle L_{1} L_{2} L_{3}, L_{1} L_{2} L_{4}, L_{1} L_{3} L_{4}, L_{2} L_{3} L_{4}\right\rangle
$$

Also consider

$$
J=\sqrt{I_{3}\left(\mathcal{A}_{\Gamma}\right)} .
$$

With Macaulay $2([7)$, observe that

$$
I_{3}\left(\mathcal{A}_{\Gamma}\right): J=\langle y+2 z, x+z\rangle \text { and } I_{3}\left(\mathcal{A}_{\Gamma}\right): J^{2}=R .
$$

From Theorem 3.2 this means that $\operatorname{hyp}(\Gamma)=3$.

Let $I=I_{1}^{m_{1}} \cap \cdots \cap I_{s}^{m_{s}}$ be the ideal of a fat point scheme. Denote $J=\sqrt{I}$ and suppose that $m_{1}=\cdots=m_{p}=m$ is the maximum multiplicity of any primary component of $I$. Then

$$
I: J^{m-1}=I_{1} \cap \cdots \cap I_{p} .
$$

In the fitting problem setup, the points with ideals $I_{1}, \ldots, I_{p}$ correspond (dually) to the hyperplanes containing $h y p(\Gamma)$ number of points of $\Gamma$. 
For our example, if we do this operation, we obtain $\langle y+2 z, x+z\rangle$, which is the ideal of the point $[-1,-2,1]$. Dually, we obtain the projective line

$$
-x-2 y+z=0
$$

which after dehomogenization gives the line in the plane of equation

$$
x+2 y=1 .
$$

Observe that the points $P_{1}, P_{3}$ and $P_{4}$ are collinear sitting on this line.

\section{ACKNOWLEDGEMENTS}

The author wishes to thank Graham Denham and Hal Schenck for useful discussions. The author is also very grateful to the anonymous referee for the important terminology correction and for improving and simplifying the proof of Proposition 3.1, which allowed us to drop the restriction on the characteristic of the field considered initially in this result as well as in Theorem 3.2 .

\section{REFERENCES}

[1] David Cox, John Little, and Donal O'Shea, Using algebraic geometry, Graduate Texts in Mathematics, vol. 185, Springer-Verlag, New York, 1998. MR.1639811 (99h:13033)

[2] Edward D. Davis and Anthony V. Geramita, Birational morphisms to $\mathbf{P}^{2}$ : an ideal-theoretic perspective, Math. Ann. 279 (1988), no. 3, 435-448, DOI 10.1007/BF01456280. MR922427 (89a:14014)

[3] Herbert Edelsbrunner, Algorithms in combinatorial geometry, EATCS Monographs on Theoretical Computer Science, vol. 10, Springer-Verlag, Berlin, 1987. MR904271 (89a:68205)

[4] David Eisenbud, Commutative algebra, With a view toward algebraic geometry, Graduate Texts in Mathematics, vol. 150, Springer-Verlag, New York, 1995. MR1322960 (97a:13001)

[5] David Eisenbud, The geometry of syzygies, A second course in commutative algebra and algebraic geometry. Graduate Texts in Mathematics, vol. 229, Springer-Verlag, New York, 2005. MR2103875 (2005h:13021)

[6] Leah Gold, John Little, and Hal Schenck, Cayley-Bacharach and evaluation codes on complete intersections, J. Pure Appl. Algebra 196 (2005), no. 1, 91-99, DOI 10.1016/j.jpaa.2004.08.015. MR2111849(2005k:14050)

[7] D. R. Grayson and M. E. Stillman, Macaulay 2, a software system for research in algebraic geometry, http://www.math.uiuc.edu/Macaulay2/.

[8] Leonidas J. Guibas, Mark H. Overmars, and Jean-Marc Robert, The exact fitting problem in higher dimensions, Comput. Geom. 6 (1996), no. 4, 215-230, DOI 10.1016/09257721(95)00020-8. MR1392311 (98c:52020)

[9] Johan P. Hansen, Linkage and codes on complete intersections, Appl. Algebra Engrg. Comm. Comput. 14 (2003), no. 3, 175-185, DOI 10.1007/s00200-003-0119-3. MR2013791 (2004j:13018)

[10] Peter Orlik and Hiroaki Terao, Arrangements of hyperplanes, Grundlehren der Mathematischen Wissenschaften [Fundamental Principles of Mathematical Sciences], vol. 300, SpringerVerlag, Berlin, 1992. MR1217488 (94e:52014)

[11] Hal Schenck, Resonance varieties via blowups of $\mathbb{P}^{2}$ and scrolls, Int. Math. Res. Not. IMRN 20 (2011), 4756-4778. MR2844937 (2012k:14075)

[12] Ştefan O. Tohăneanu, Lower bounds on minimal distance of evaluation codes, Appl. Algebra Engrg. Comm. Comput. 20 (2009), no. 5-6, 351-360, DOI 10.1007/s00200-009-0102-8. MR:2564409(2011c:13027)

[13] Ştefan O. Tohăneanu, On the de Boer-Pellikaan method for computing minimum distance, J. Symbolic Comput. 45 (2010), no. 10, 965-974, DOI 10.1016/j.jsc.2010.06.021. MR2679386 (2011g:94074)

[14] Ştefan O. Tohăneanu, The minimum distance of sets of points and the minimum socle degree, J. Pure Appl. Algebra 215 (2011), no. 11, 2645-2651, DOI 10.1016/j.jpaa.2011.03.008. MR2802154 (2012e:13028) 
[15] Ştefan O. Tohaňeanu and Adam Van Tuyl, Bounding invariants of fat points using a coding theory construction, J. Pure Appl. Algebra 217 (2013), no. 2, 269-279, DOI 10.1016/j.jpaa.2012.06.004. MR2969252

[16] Wolmer V. Vasconcelos, Computational methods in commutative algebra and algebraic geometry, Algorithms and Computation in Mathematics, vol. 2, Springer-Verlag, Berlin, 1998. With chapters by David Eisenbud, Daniel R. Grayson, Jürgen Herzog and Michael Stillman. MR:1484973(99c:13048)

Department of Mathematics, The University of Western Ontario, London, On N6A 5B7, CANADA

E-mail address: stohanea@uwo.ca

$U R L:$ http://www . math. uwo.ca/ stohanea/

Current address: Department of Mathematics, University of Idaho, 875 Perimeter Drive, MS1103, Moscow, Idaho 83844-1103

E-mail address: tohaneanu@uidaho.edu 